\title{
Research and Simulation of fault detection method for small electronic equipment
}

\author{
Zeng Zhaomin
}

Sichuan Information Technology College 608040

Keywords: fault detection; feature extraction; wavelet analysis;

\begin{abstract}
: in the process of studying fault detection method for small electronic devices, when using current algorithm for small electronic equipment fault detection, too many constraints results in incomplete detection, large error and time consuming. To this end, a fault detection method for small electronic devices based on improved immune algorithm is proposed. The method fusing in wavelet analysis technology extracts the fault information of small electronic equipment detection system first, and then uses the wavelet modulus maximum to map the transient response of the fault state. On the basis of the risk theory model, the antigen collection and antibody groups in real-time fault detection system of small electronic equipment are matched with the obtained fault feature information one by one, and a multi threshold is set up to determine whether it is a fault signal, thus, a small electronic equipment fault detection is completed efficiently. The experimental results show that the method of fault detection based on small electronic devices is highly effective.
\end{abstract}

\section{Introduction}

With the development of electronic information technology, small electronic devices have been used in the construction of various industries due to its small size, light weight and compact structure [1-3]. However, small electronic devices are susceptible to environmental impact (such as humidity, mold, salt spray, etc.) and results in a series of failures, and the difficulty of detection is large [4-6]. Therefore, how to detect the fault of small electronic devices is the main problem to be solved in this field [7-9]. At present, the main method of fault detection is based on genetic algorithm, ant algorithm and neural network algorithm [10]. Among them, which often used in the detection of small electronic equipment is based on ant algorithm, but the algorithm has too many constraints, the detection is not comprehensive, and the error is large and time-consuming.

To this end, a fault detection method for small electronic devices based on improved immune algorithm is proposed. The method fusing in wavelet analysis technology extracts the fault information of small electronic equipment detection system first, and then uses the wavelet modulus maximum to map the transient response of the fault state. On the basis of the risk theory model, the antigen collection and antibody groups in real-time fault detection system of small electronic equipment are matched with the obtained fault feature information one by one, and a multi threshold is set up to determine whether it is a fault signal, thus, a small electronic equipment fault detection is completed efficiently. The experimental results show that the method of fault detection based on small electronic devices is highly effective.

\section{Fault detection principle of small electronic devices}

During fault detection process for small electronic devices, extract non-stationary time-varying signal of small electronic device detection system and respond, set the appropriate initial threshold, and compare with the extracted fault signal, to determine whether it is a fault signal of small electronic devices. Detailed steps as below:

During fault detection process for small electronic devices, set $d_{i j}$ indicating the detection attribute of detection point $t_{j}$ to a fault condition $f_{i}$, the value of $d_{i j}$ is $[\tilde{0} k-1]$, using the following formula can draw detection relevant moments of the small electronic device represented by $D$ :

$$
D_{(m+1) \times n}=\left[\begin{array}{lll}
d_{01} & d_{02} & d_{0 n} \\
d_{11} & d_{12} & d_{1 n} \\
d_{m 1} & d_{m 2} & d_{m m}
\end{array}\right]
$$

In the above formula, the number of rows of the matrix $D$ represents the number of detected fault 
conditions of small electronic devices, the number of columns of $D$ represents the number of detected vector.

\section{Optimized failure detection principle of small electronic devices}

3.1 extraction of fault signal of small electronic devices

In optimized fault detection process of small electronic devices, combining with wavelet analysis technology to extract the fault information of small electronic equipment detection system first, and then uses the wavelet modulus maximum to map the transient response of the fault state. Concrete steps as below:

(1) for small-sized electronic device failure signal process $m$ layer wavelet packet decomposition, the signal characteristics of $2^{m}$ frequency components $\mathrm{xx}\left(X_{1}, X_{2 \ldots}\right)$ are extracted from low frequency to high frequency at $m\left(X_{1}, X_{2 \ldots}\right)$ layer, expressed by the following equation:

$$
\frac{m}{\left(X_{1}, X_{2 \ldots}\right)}=\frac{2^{m}}{m \times\left(X_{1}, X_{2 \ldots}\right)}
$$

(2) reconstructing wavelet packet decomposition coefficients, and extracting fault signal of small electronic devices at each frequency band range. $S_{1}$ expresses reconstructed signal of $X_{1}, S_{2}$ represents the reconstructed signal of next, and so on. The exothermic total signal $S$ of small electronic device testing system can be expressed as:

$S=S_{1}+S_{2}$

(3) In optimized fault detection process of small electronic devices, the total energy of small electronic devices signal at each band is calculated. Let $S_{i}(i=1,2, \ldots)$, the corresponding energy $E_{i}(i=1,2, \ldots)$, then the following equation can have the mapping relationship from system energy to fault:

$$
E_{i}=\int|s i(t)|^{2} d t=6_{k=1}^{n}\left|x_{i, k}\right|^{2}
$$

In the formula, $x_{i, k}$ on behalf of the discrete points amplitude of reconstructed signal $S_{i}$ of small electronic devices detection system, $i=0,1,2, k=1,2,3, n$ is signal sampling points.

(4) In optimized fault detection process of small electronic devices, construct fault feature vector used each band energy as elements. Expressed by the following formula

$E=\left(E_{1}, E_{2}, E_{3}\right)$

(5) In optimized fault detection process of small electronic devices, wavelet transform modulus maxima corresponds to a singular point of small electronic device detection system signals, the singular point of small electronic devices signal is located by detecting modulus maxima. Assuming a modulus maxima $T_{j}$ at the scale $j$, then modulus maxima of each scale in respective positions may constitute a sequence $\left\{T_{j}\right\}, \quad j=1,2,3$, when $j$ is small, have the following approximation:

$a_{j}=W_{2}^{j} f\left(x_{0}\right) \approx A 2^{j T}$

In optimized fault detection process of small electronic devices, according to the following formula can acquire transient response characteristics of small electronic device detection system fault condition:

$\log _{2} a_{j}=\log _{2} A+j T$

\subsection{Implementation of optimized failure detection principle of small electronic devices}

Under the dangerous theoretical model, the signals received by electronic equipment failure real-time detection system in the immune recognition process is divided into three types: danger signal (signal0); antigen-presenting signal (signal1); costimulatory signal (signal2); the antigen group and antibody collection in small electronic real-time fault detection system are matched to obtained fault features information one by one, and set multiple thresholds to determine whether the fault signal. Concrete steps as below: 
(1) Set the initial state of each threshold value of electronic equipment failure real-time detection system, and the signal (signal0), (signal1), (signal2) are cleared.

(2) Input string-constituted antibody group in electronic equipment failure real-time detection system. It can be expressed by the following equation:

$b_{g}=\left\{v_{b 1}, v_{b 2}, v_{b 3}, \ldots, v_{b n}\right\}$

In the above formula, $b_{g}$ on behalf of the real-time fault detection antibody group.

(3) Enter the new antigen group in small electronic device real-time fault detection system, can be expressed using the following formula:

$$
a_{g}=\left\{v_{a 1}, v_{a 2}, v_{a 3}, \ldots, v_{a n}\right\}
$$

In the above formula, $a_{g}$ represents the new antigen collection.

(4) The antigen group and antibody collection in small electronic real-time fault detection system are matched to obtained fault features information one by one, used to calculate affinity of fault real-time detection system. Expressed using the following formula:

$$
\operatorname{aff} \operatorname{inity}\left(a_{g}, b_{g}\right)=\frac{1}{1+\sum_{i=1}^{n} \sqrt{\sum_{j=1}^{M}\left(v_{a i j}-v_{b i j}\right)}}
$$

In the above formula, aff in ity represents affinity between antigen $a_{g}$ and antibody $b_{g}$ in electronic equipment failures real-time detection.

(5) the affinity of fault detection system calculated from step 4 compared with a threshold value, if exceeds the threshold value, it indicates that the input is abnormal, so that (signal1) $=1$, otherwise $($ signal0) $=0$ skip to Step 3 .

\section{Experiments and simulation}

In order to prove the effectiveness of proposed fault detection method based on improved immune algorithm for small electronic devices, an experiment is needed. Build a fault detection virtual experimental platform utilizing Labview, under the different number of experiments, the improved algorithm and the traditional algorithms were utilized separately for fault detection test of small electronic devices, accuracy, detection time, detection error rate of two algorithms fault extraction feature were compared, used to measure the detection effectiveness of different algorithms. Comparative results are shown in Table 1 and Table 2.

Table 1 the overall performance of the traditional fault detection algorithm for small electronic devices

\begin{tabular}{cccc}
\hline $\begin{array}{c}\text { The number of } \\
\text { experiments }\end{array}$ & $\begin{array}{c}\text { Accuracy of } \\
\text { extracted fault } \\
\text { signature }(\%)\end{array}$ & $\begin{array}{c}\text { Detection time } \\
(\mathrm{S})\end{array}$ & $\begin{array}{c}\text { The detection error } \\
\text { rate } \\
(\%)\end{array}$ \\
\hline 10 & 76 & 2.9 & 0.6 \\
20 & 73 & 2.9 & 0.6 \\
30 & 76 & 2.6 & 0.6 \\
40 & 76 & 2.6 & 0.8 \\
\hline
\end{tabular}

Table 2 the overall performance of the improved fault detection algorithm for small electronic devices

\begin{tabular}{cccc}
\hline $\begin{array}{c}\text { The number of } \\
\text { experiments }\end{array}$ & $\begin{array}{c}\text { Accuracy of } \\
\text { extracted fault } \\
\text { signature }(\%)\end{array}$ & $\begin{array}{c}\text { Detection time } \\
(\mathrm{S})\end{array}$ & $\begin{array}{c}\text { The detection error } \\
\text { rate } \\
(\%)\end{array}$ \\
\hline 10 & 96 & 1.7 & 0.1 \\
20 & 96 & 1.6 & 0.1 \\
30 & 95 & 1.7 & 0.1 \\
40 & 95 & 1.7 & 0.1 \\
\hline
\end{tabular}

From Table 1 and Table 2 can be described, the fault detection method based on small electronic 
devices has high detection effectiveness, and complete the fault detection for small electronic devices efficiently.

\section{Conclusion}

Since using current algorithm for small electronic equipment fault detection, too many constraints results in incomplete detection, large error and time consuming. To this end, a fault detection method for small electronic devices based on improved immune algorithm is proposed. The method fusing in wavelet analysis technology extracts the fault information of small electronic equipment detection system first, and then uses the wavelet modulus maximum to map the transient response of the fault state. On the basis of the risk theory model, the antigen collection and antibody groups in real-time fault detection system of small electronic equipment are matched with the obtained fault feature information one by one, and a multi threshold is set up to determine whether it is a fault signal, thus, a small electronic equipment fault detection is completed efficiently. The experimental results show that the method of fault detection based on small electronic devices is highly effective.

\section{Reference:}

[1] Yang Xiaofang, Li Xuehua. Diagnosis and some simple maintenance method for electronic equipment intermittent failures [J] Electronic world, 2013, (24): 121-121.

[2] Yi Shuangquan. Fault detection methods and techniques analysis for electronic circuit [J] Consumer electronics, 2013, (16): 51-51.

[3] Liu Yixiao. Brief talk on medical electronic equipment fault diagnosis [J] Electronic Technology and Software Engineering, 2014, (5): 128-128.

[4] Xie Min. Simulation on Power Electronic Circuit Fault Diagnosis Method [J] Computer Simulation, 2013, 30 (12): 372-375.

[5] Pang Liwei, Xue Wenhu, Yang Hongli. Chaos adaptive Particle Swarm Optimization for fault detection [J]. Computer and Digital Engineering, 2014, 42 (3): 407-411.

[6] Chen Chen, Hu Weiwei, Sun Yufeng, et al. Electronic watt-hour meter fault detection method based on BP-AdaBoost [J] Electronics Optics \& Control, 2013, 20 (4): 72-76.

[7] Xing Tong, Chen Jinbao, Zhai Guofu, et al. Remainder automatic detection system of onboard electronic equipment [J] Electronic Measurement and Instrument, 2013, 27 (4): 359-365.

[8] Zhang Ya-ni, Hu Qing. Thermal design of an airborne electronic equipment [J] Modern electronic technology, 2013, 36 (3): 151-153.

[9] Lv Jingfeng, Chen Lingxiang. Electromagnetic compatibility of Electronic devices structures design [J] Electronic world, 2013, (12): 163-163.

[10] Liu Wangping. Brief talk on grounding problem of sophisticated electronic equipment and countermeasures [J] Electronic world, 2014, (2): 103-104. 